\title{
Comparative analysis of the honeycomb and thin-shell space antenna reflectors
}

\author{
Sergey V. Reznik ${ }^{1, *}$, and Andrey D. Novikov ${ }^{1}$ \\ ${ }^{1}$ Bauman Moscow State Technical University, 105005 Moscow, Russia
}

\begin{abstract}
Parabolic three-layered reflectors from polymer composite materials with the aluminium honeycomb fillers became widely used in space communication systems in the past decades. There are technological possibilities for creating reflectors in the form of thin-walled ribbed shell with the lower linear density than that of the three-layered structures. The paper presents the results of the temperature and stress-strain analysis for the two types of structures, which could help to select the variant with the best performance characteristics.
\end{abstract}

\section{Introduction}

The state-of-the-art space antenna reflectors are essentially parabolic shells with various types of reinforcement that enable the required stiffness. The most frequent design layout is a three-layered shell with the honeycomb reinforcement, less frequent is a thin shell with the reinforcing ribs over the convex surface [1]. The common features for all structures are, firstly, the requirement for the high shape and dimensional stability under condition of varying temperature as a result of the spacecraft entering the Earth shadow and, secondly, low linear density (the mass in relation to the surface area). The design of the reflectors with the honeycomb filler meets the most stringent requirements with the regard to the shape and dimensional stability, while having a relatively small linear density in the range of 3.5-5.0 kg/m $\mathrm{m}^{2}$ [2]. Sources [3] and [4] presented the results of simulating the thermal and stress-strain behavior of several reflector variants with the concave shell ribbed reinforcement for the geostationary Earth orbit (GEO) conditions, which proved the possibility of the designs with the small linear density and high stiffness [4]. After the analysis of various reinforcement patterns [5], the "five-point star" pattern was singled out, as being superior to the other variants with respect to stiffness and linear density.

This research aims to compare the characteristics of the space antenna reflector layouts with the honeycomb filling and ribbed reinforcement. In order to achieve this aim, the following objectives were fulfilled consecutively:

- Geometric models were developed;

- Thermal physical characterisctics of the honeycomb filling were determined;

- Temperature and stress-strain state at GEO was determined.

*Corresponding author: sreznik@bmstu.ru 


\section{Geometric models development}

The comparative analysis involved building geometric models of the design with the honeycomb filling (Fig. 1) and the ribbed reinforcement (Fig. 2). The shells in the both variants were made from carbon fibre composite.

The temperature and stress-strain simulation was performed using the finite-element method in the Siemens NX PLM software package. The carbon fibre composite was assumed to have the following thermal physical and mechanical properties: thermal conductivity coefficient $1000 \mathrm{~J} /(\mathrm{kg} \cdot \mathrm{K})$; density $1550 \mathrm{~kg} / \mathrm{m}^{3}$; emissivity 0.85 ; absorptivity 0.735; CLTE 5.27·10 $0^{-7}$, Young's modulus $140 \mathrm{GPa}$, Poisson ratio 0.3 .

In the honeycomb filled design each carbon fibre composite shell was $0.6 \mathrm{~mm}$ thick, the honeycomb layer was $25 \mathrm{~mm}$ thick.

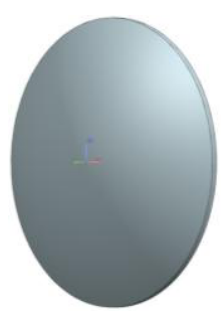

Fig. 1. Three-layered construction with the honeycomb filling.

The ribbed reinforcement design was essentially a smooth parabolic shell $0.4 \mathrm{~mm}$ thick with flat ribs, $90 \mathrm{~mm}$ high and $0.6 \mathrm{~mm}$ thick.

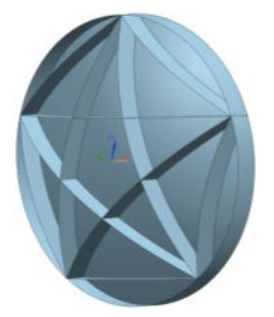

Fig. 2. Ribbed reinforcement design.

\section{Honeycomb filler characterization}

For the finite-element analysis of the thermal state, the information about the thermal physical and mechanical properties of the honeycomb fillers is essential. The unified reference data for the honeycomb materials are not available, which necessitates conducting thermal physical and mechanical characterization for each design layout.

The mechanical characteristics of the honeycomb materials were determined by menas of the e-Xstreme Digimat 6.0. The input data included the $\mathrm{AMr}-2$ alloy properties, in particular, Young's modulus $71 \mathrm{GPa}$, density $2680 \mathrm{~kg} / \mathrm{m}^{3}$; the honeycomb characteristics: hexagonal pattern, $0.015 \mathrm{~mm}$ wall thickness, $25 \mathrm{~mm}$ filler height. The mechanical properties values for the honeycomb material are given in Tables 1 and 2 .

Table 1. Young's modulus of the honeycomb material.

\begin{tabular}{|c|c|}
\hline Axis & Young's modulus, MPa \\
\hline$x$ & 0.035 \\
\hline$y$ & 0.035 \\
\hline$z$ & 484.989 \\
\hline
\end{tabular}


Table 2. Poisson's ratios and shear modulus of the honeycomb material.

\begin{tabular}{|c|c|c|}
\hline Plane & Poisson's ratio & Shear modulus, MPa \\
\hline$x y$ & 0.99 & 0.0087 \\
\hline$y z$ & 0.33 & 91.1633 \\
\hline$z x$ & 0.33 & 91.1633 \\
\hline
\end{tabular}

The thermal physical properties in the longitudinal direction were determined using a 3D model of the honeycomb elementary cell, with $0.015 \mathrm{~mm}$ wall thickness and $25 \mathrm{~mm}$ height. Hexagonal polygonal bodies $0.6 \mathrm{~mm}$ thick were added above and below it to simulate the carbon fibre shell. After that a finite-element model was created and the boundary conditions were specified in the form of temperature and radiative heat transfer inside the cell.

The Siemens NX Nastran was used to conduct the finite-element analysis for 15 calculating cases simulating various operating temperatures at $20{ }^{\circ} \mathrm{C}$ interval: from minus $120{ }^{\circ} \mathrm{C}$ to plus $160{ }^{\circ} \mathrm{C}$. The heat flux density through the elementary honeycomb cell was determined in accordance with Fourier law:

$$
q=-\lambda_{\Im \phi} \frac{T_{1}-T_{2}}{\delta_{\Sigma}}
$$

where $q$ is the heat flux density; $\lambda_{\text {eff }}$ is the effective heat conductivity, $T_{1}$ is the temperature on the upper shell surface, $T_{2}$ is the temperature on the lower shell surface.

Temperatures $T_{1}, T_{2}$ for each case were specified with a $2{ }^{\circ} \mathrm{C}$ difference. For example, for the $60{ }^{\circ} \mathrm{C}$ case the temperature $T_{1}$ constituted $59{ }^{\circ} \mathrm{C}, T_{2}-61{ }^{\circ} \mathrm{C}$. After the heat density flux was determined, the honeycomb material thermal conductivity was estimated

$$
\begin{gathered}
\delta_{\Sigma}=\delta_{1}+\delta_{2}+\delta_{3} ; \\
\frac{\delta_{\Sigma}}{\lambda_{3 \phi}}=\frac{\delta_{1}}{\lambda_{1}}+\frac{\delta_{2}}{\lambda_{2}}+\frac{\delta_{3}}{\lambda_{3}} .
\end{gathered}
$$

where $\lambda_{1}, \lambda_{3}$ are the shell thermal conductivities; $\lambda_{2}$ is the filler thermal conductivity.

The estimation of the thermal efficiency in the transverse direction employed the layout similar to that for the longitudinal direction. The thermal load conditions were identical to the estimation of the longitudinal thermal conductivity.

The thermal conductivity of the honeycomb filler in the longitudinal and transverse directions is presented in Fig. 3 .

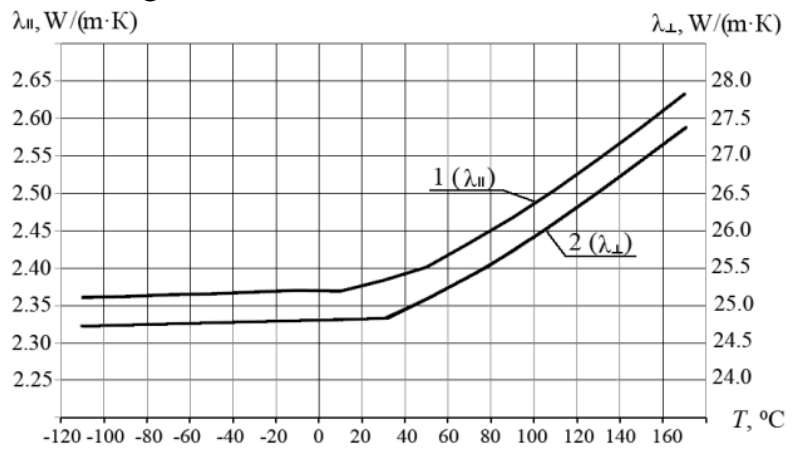

Fig. 3. Thermal efficiency of the honeycomb material as a function of temperature, longitudinal (1) and transverse (2) directions. 


\section{Determining the stress-strain behavior of the honeycomb filler structure}

The temperature gradient was determined by means of Space Systems Thermal solver from the Siemens NX software package. The research involved analyzing 24 moments of operation in the GEO. The largest gradient coincided with the moment when the reflector axis of rotation was at $150^{\circ}$ relative to the Earth-Sun axis. The thermal calculation data were used as the reference data for stress-strain calculation. The simulation results are presented in Fig. 4.

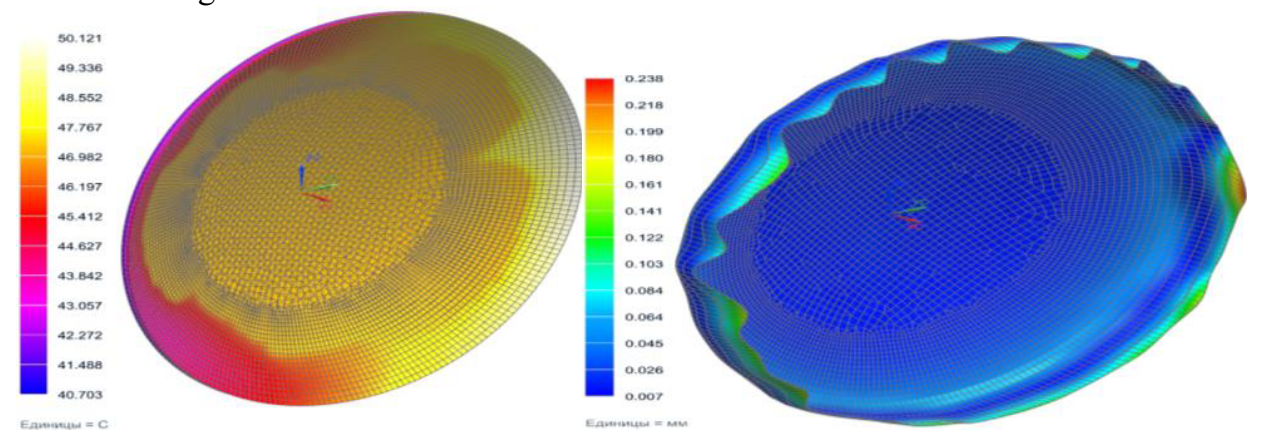

Fig. 4. Thermal (left) and stress-strain (right) behavior of the three-layered structure at the moment, when the reflector axis of rotation was at $150^{\circ}$ angle the Earth-Sun axis at GEO.

\section{Determining the stress-strain behavior of the ribbed reinforcement structure}

To estimate the stress-strain behavior of the ribbed reinforcement structure, 24 moments of the work at GEO were analyzed. The largest temperature gradient coincided with the moment when the reflector axis of rotation was at $120^{\circ}$ relative to the Earth-Sun axis. The thermal calculation data were used as the reference data for stress-strain calculation. The simulation results are presented in Fig. 5.

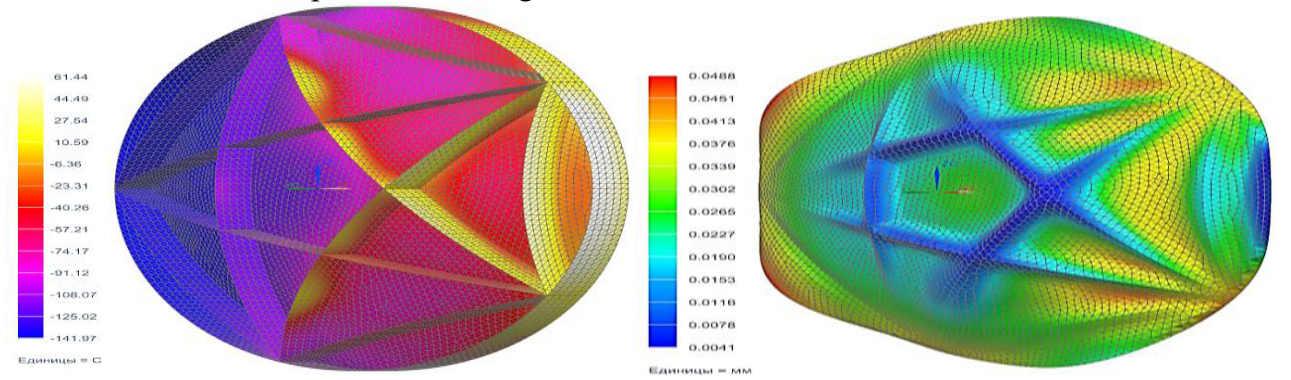

Fig. 5. Thermal (left) and stress-strain (right) behavior of the ribbed reinforcement structure at the moment, when the reflector axis of rotation was at $120^{\circ}$ angle to the Earth-Sun axis at GEO.

\section{Analysis}

Table 3 presents the comparison of the design layout characteristics under investigation. 
Table 3. Comparative characteristics of the design layouts.

\begin{tabular}{|c|c|c|c|}
\hline Layout & $\begin{array}{c}\text { Temperature gradient, } \\
{ }^{\circ} \mathbf{C}\end{array}$ & $\begin{array}{c}\text { Maximum } \\
\text { displacement, } \mathbf{m m}\end{array}$ & Mass, kg \\
\hline $\begin{array}{c}\text { Three-layered } \\
\text { structure }\end{array}$ & 9.42 & 0.238 & 2.6 \\
\hline $\begin{array}{c}\text { Ribbed reinforcement } \\
\text { structure }\end{array}$ & 184.54 & 0.049 & 1.224 \\
\hline
\end{tabular}

As is evident from Table 3 the honeycomb filler structure is characterized by a more uniform distribution and a lower temperature gradient. The three-layered structure had 0.238 maximum displacement, while the mean displacement across the surface is in the range of 0.04 to $0.06 \mathrm{~mm}$. The maximum displacement for the ribbed reinforcement structure constituted 0.049 . However, the ribbed reinforcement layout has a clear advantage with the mass twice as low as that of the honeycomb filler layout. Therefore, the ribbed reinforcement layout is selected for the further development.

Some results of this work were obtained in the framework of the project №1864 under the state commission №2014/104 for the public research activity as part of the commission by the Ministry of education and science of the Russian Federation.

\section{References}

1. S.V. Reznik, P.V. Prosuntsov, A.V. Azarov, J. Eng. Phys. 88, 674 (2015)

2. Pfeiffer, K.E. Highly stable CFPR antenna reflectors HPS GmbH (2007)

3. S.V. Reznik, P.V. Prosuntsov, A.V. Azarov, J. Eng. Phys. 88, 945 (2015)

4. P.V. Prosuntsov, S.V. Reznik, K.V. Mikhailovsky, A.D. Novikov, IOP Conf. 153, $012012(2016)$

5. S.V. Reznik, P.V. Prosuntsov, A.D. Novikov, Proceed. XV Minsk International Heat and Mass Transfer Forum 2, 423 (2016)

6. GOST 21631-76. Sheets of aluminum and aluminum alloy (Standarts Publishing House, 1993) 